\title{
Quantifying Atomic-Scale Quantum Dot Superlattice Behavior Upon in situ Heating
}

\author{
Michelle A. Smeaton ${ }^{1}$, Daniel M. Balazs², Tobias Hanrath², and Lena F. Kourkoutis ${ }^{3,4, *}$ \\ 1. Department of Materials Science \& Engineering, Cornell University, Ithaca, NY, USA. \\ 2. School of Chemical \& Biomolecular Engineering, Cornell University, Ithaca, NY, USA. \\ 3. School of Applied \& Engineering Physics, Cornell University, Ithaca, NY, USA. \\ 4. Kavli Institute for Nanoscale Science, Cornell University, Ithaca, NY, USA. \\ * Corresponding author: lena.f.kourkoutis@cornell.edu
}

The recent proliferation of synthesis methods for quantum dots (QDs) with precisely controlled shapes, sizes and compositions has made possible a new class of materials comprised of superlattices (SLs) of epitaxially connected QDs called quantum dot solids (QDS). The combination of precisely controlled, quantum confined structures and increased coupling between neighboring QDs mean these materials represent exciting opportunities for advancing optoelectronic devices and realizing exotic electronic phenomena [1]. However, the complex interplay of particle diffusion, assembly, and attachment during QDS formation has proved a formidable barrier to achieving ordered SLs with defect free epitaxial connections $[2,3]$. Heating QDS may provide one solution to these problems by annealing out defects and promoting surface diffusion of atoms to form or strengthen epitaxial connections [4]. While heating inside a TEM has been used to track reactions, grain growth and atomic-scale reorganization, quantifying the behavior of QDS upon heating requires large fields of view at atomic resolution to access a statistically meaningful number of QDs, which poses new challenges.

Here, we use a DENS Solutions MEMS heating holder in combination with aberration-corrected HAADFSTEM and image analysis to assess the effects of heating on the local order of QDS over extended fields of view. We analyze PbS QDs self-assembled into square SLs and epitaxially connected along the $<100>$ directions (Fig. 1a). Localized heating provided by the MEMS device minimizes thermal drift and maintains stage stability such that a region of interest can be tracked throughout a heating cycle and images can be acquired at atomic-resolution over $100 \mathrm{~nm}$ fields of view. From these images, we extract QD positions and orientations using automated image analysis tools developed in Python (Fig. 1b-f).

Analysis of individual images is straightforward; however, additional challenges arise when comparing images of a region of interest acquired before and after heating. In particular, though STEM images appear drift free visually, they may contain shear artifacts due to minor sample drift during acquisition, which become obvious when aligning before and after images or when overlaying their FFTs (Fig. 2a). Shear due to sample drift can be minimized by taking fast acquisition stacks and carefully aligning the images using image registration. Assuming drift occurs at a constant rate during acquisition, the resulting shear angle can be estimated and corrected in post processing (Fig. 2b), allowing the before and after images to be accurately aligned for direct comparison. Another challenge, requiring development of new analysis techniques, arises from variation in the number and size of connections between a QD and its nearest neighbors, which skews calculation of the QD centroid (Fig. 2c-d). This skew can significantly impact analysis of local SL order and has to be corrected for in order to quantify subtle differences in QD position.

Figure 3 shows the evolution of out-of-plane QD orientations in a QDS sample beginning with its as-

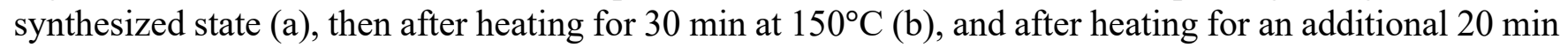
at $200^{\circ} \mathrm{C} \mathrm{(c).} \mathrm{Our} \mathrm{analysis} \mathrm{shows} \mathrm{a} \mathrm{progressive} \mathrm{increase} \mathrm{in} \mathrm{the} \mathrm{number} \mathrm{of} \mathrm{correctly} \mathrm{oriented}(<100>)$ QDs and a corresponding decrease in the number of slightly mis-oriented $(<\ln 0>$ and $<1 \mathrm{nn}>$ ) QDs upon heating, indicating the potential of this method to anneal out defects in imperfect QDS [5]. 
[1] E. Kalesaki et al, Phys. Rev. X 4 (2014), p. 011010.

[2] K. Whitham et al, Nature Materials 15 (2016), p. 557.

[3] B. Savitzky et al, Nano Lett. 16 (2016), p. 5714.

[4] J. C. Ondry, M. R. Hauwiller, and A. P. Alivisatos, ACS Nano 12 (2018), p. 3178.

[5] This work is supported by DOE (DE-SC0018026) and NSF (DMR-1719875, MRI-1429155).
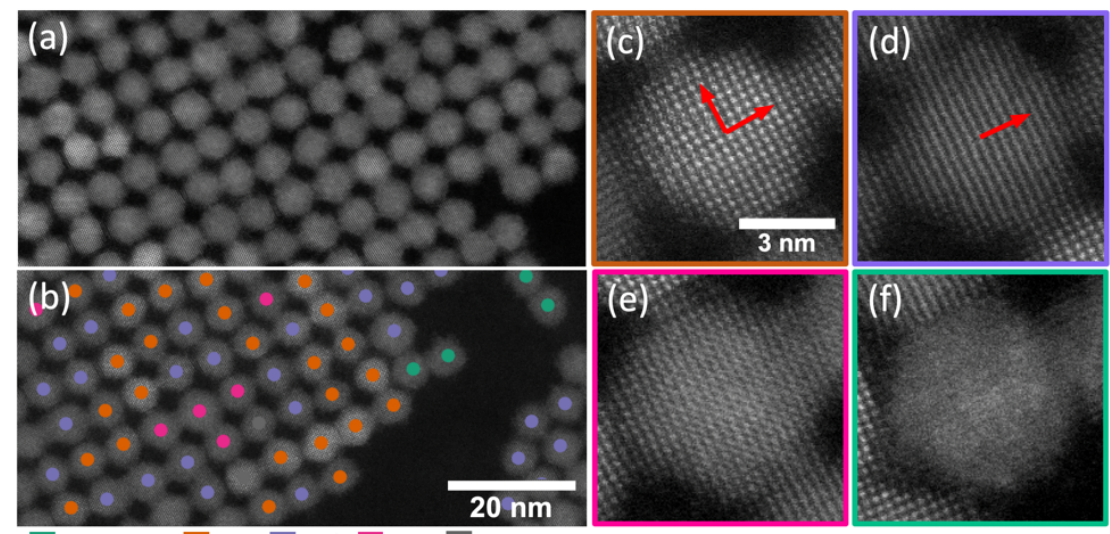

Figure 1. (a-b) A STEM image of PbS QDs forming a monolayer square SL epitaxially connected via the $<100>$ facets acquired using a DENS Solutions MEMS heating holder. The extracted out-of-plane orientations are overlaid in (b), and representative QDs having $<100\rangle,\langle 1 \mathrm{n} 0\rangle,<1 \mathrm{nn}\rangle$ and misaligned orientations are shown in (c-f) respectively with their in-plane orientations overlaid.

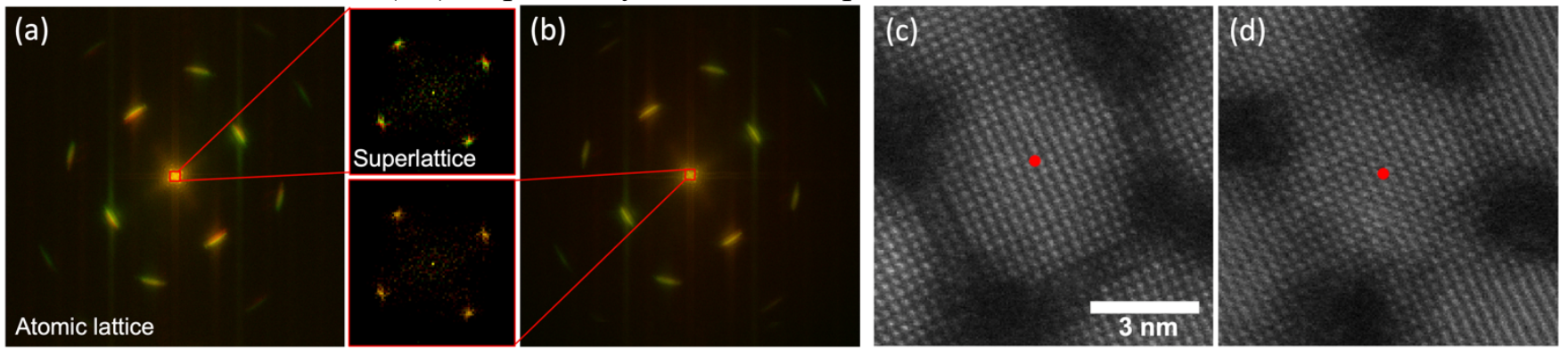

Figure 2. (a-b) Overlaid FFTs of images acquired before (red) and after (green) one heating cycle. Shearing effects are evident in the raw data (a) by the offset in both the atomic lattice and superlattice (inset) Bragg spots. The shear angle was identified and corrected in post processing (b), resulting in perfectly aligned Bragg spots. (c-d) Images of one QD (c) exhibiting variation in epitaxial connection width resulting in a skewed centroid, and one QD (d) with even connections showing an accurate centroid.

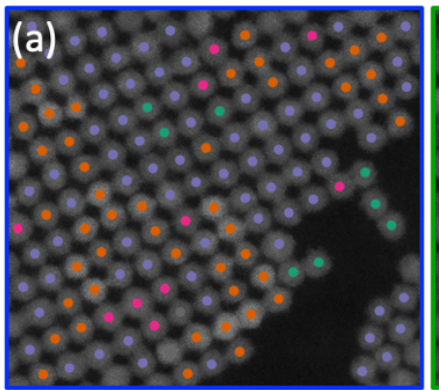

misaligned $\square 100$

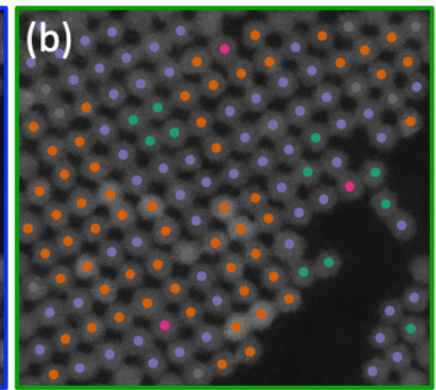

$1 \mathrm{n} 0$

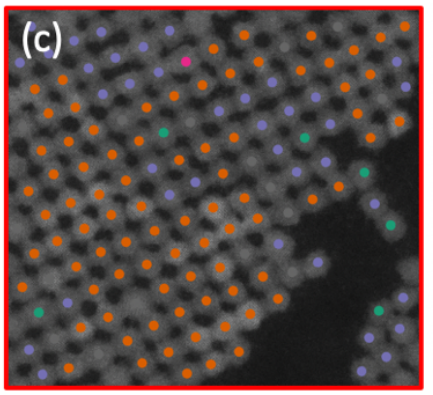

unknown

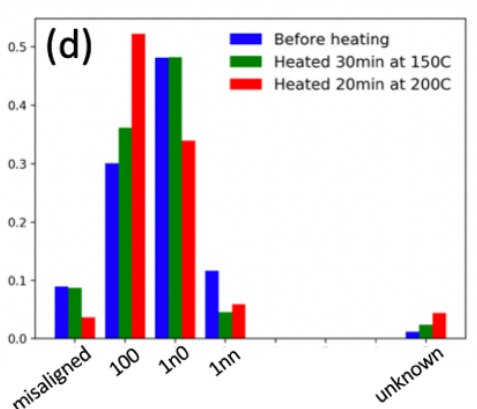

Figure 3. (a-c) Images of the same region of interest before heating (a), after heating for $30 \mathrm{~min}$ at $150^{\circ} \mathrm{C}$ (b), and after heating an additional $20 \mathrm{~min}$ at $200^{\circ} \mathrm{C}$ (c) with out-of-plane orientations of QDs overlaid showing a clear increase in the number of correctly oriented $<100>$ QDs. (d) Out-of-plane orientations for the same three heating conditions compiled for $\sim 400$ QDs. 